\title{
E53 PREDICTION UNDER UNCERTAINTY IN RESERVOIR MODELING
}

\author{
Mike Christie', Sam Subbey ${ }^{1}$, Malcolm Sambridge ${ }^{2}$ \\ ${ }^{1}$ Institute of Petroleum Engineering, Heriot-Watt University, Edinburgh, EH14 4AS, UK \\ ${ }^{2}$ Institute of Advanced Studies, Australian Nat. University, Canberra, ACT 0200, Australia
}

\begin{abstract}
Reservoir simulation is routinely employed in the prediction of reservoir performance under different depletion and operating scenarios. Usually, a single history matched model, conditioned to production data, is obtained. The model is then used to forecast future production profiles. Since the history match is non-unique, this is essentially an inverse problem. Hence the forecast production profiles are uncertain, although this uncertainty is not usually quantified.

This paper presents a new approach for generating uncertain reservoir performance predictions and quantifying the uncertainty associated with forecasting future performance. Firstly, we generate multiple reservoir realizations using a new stochastic algorithm. This involves adaptively sampling the model parameter space using an algorithm, which biases the sampling towards regions of good fit. Using the complete ensemble of models generated, we resample from the posterior distribution and quantify the uncertainty associated with forecasting reservoir performance, in a Bayesian framework.

To demonstrate the strength of the method in performance prediction, we use an upscaled model to history match fine scale data. We then forecast the fine grid performance using the maximum likelihood model and quantify the uncertainty associated with the predictions. We demonstrate that the maximum likelihood model is highly accurate in reservoir performance prediction.
\end{abstract}

This method differs from other methods for generating multiple reservoir realizations in the following way. Rather than seeking a single global optimum, the algorithm selectively samples parameter space to derive an ensemble of models. These models share the common property of fitting the observed data to some degree of accuracy. This approach is reasonable since the inverse problem is ill-posed. In contrast to other stochastic methods, the algorithm performs a guided search in parameter space by using information derived from the complete ensemble of previously generated models. Hence no external directionality is imposed on the search process.

In Bayesian analysis, the posterior probability distribution characterizes the uncertainty in the model parameters estimated from an ensemble of models. Correctly sampling from this distribution is therefore essential for accurate quantification of forecasted reservoir performance. The Neigbourhood algorithm utilizes the nearest neighbor property of the Voronoi cells, together with a Markov Chain Monte Carlo algorithm, in correctly sampling from the posterior probability distribution.

\section{Introduction}

Petroleum reservoir data is inherently uncertain. The field information is usually sparse and noisy. Part of the data is obtained from cores $\left(\sim 10^{-17}\right.$ of the reservoir volume $)$ collected at a finite

$8^{\text {th }}$ European Conference on the Mathematics of Oil Recovery - Freiberg, Germany, 3 - 6 September 2002 
set of wells. The data may also consist of time averaged responses over large scales or derived from an incomplete knowledge of the subsurface geology. The standard procedure for reducing the uncertainty is by constraining the model to data representative of the chosen recovery scheme, i.e., dynamic data, in the form of oil, water and gas production rates, as well as pressure. In contrast to the static data (e.g., geometry and geology) obtained prior to the inception of production, these data are a direct measure of the reservoir response to the recovery process in application. This process of incorporating dynamic data in the generation of reservoir models is known as history matching.

History matching involves adjusting model parameters, with the aim of obtaining a model output, which is as close to the history (dynamic) data as possible. Mathematically, we want to determine a spatial distribution $r(\boldsymbol{x})$, and a set of model parameters $\boldsymbol{p}$, given the history data $\Gamma_{\mathrm{h}}$, such that $\Gamma_{\mathrm{s}}-\Gamma_{\mathrm{h}} \approx 0$, where $\Gamma_{\mathrm{s}}=\mathrm{f}(r, p)$ is the simulated/model output, and $x \equiv(x, y) \in R^{2}$, $x \equiv(x, y, z) \in R^{3}$, for two and three dimensional consideration of spatial data, respectively. That is, finding a zero to a multivariate time varying function, namely, the reservoir simulator. In the simplest case, one assumes the geology to be true, i.e., $r(\boldsymbol{x})$ is known. The history matching problem then reduces to determining $\boldsymbol{p}$. The problem is non-linear, inverse and ill-posed, since it does not fulfill Hadamard's classical requirements of existence, uniqueness and stability, under data perturbations, of a solution [6]. Thus instead of a single history-matched model, there exist a family of models, all of which will match the observed data to some degree of accuracy. The fundamental task is how to generate this family of solutions by exhaustive exploration of parameter space. Exploring a multidimensional space is non-trivial. Deterministic $[7,8,12]$ and stochastic $[11,13,16]$ algorithms for history matching have been reported in the literature.

The most efficient deterministic methods reported are the gradient methods such as Steepest Descent, Gauss-Newton and Levenberg-Marquardt algorithms. The methods involve calculating sensitivity parameters and require the differentiation of the mathematical model with respect to the model parameters. These sensitivity parameters are then used to construct a Hessian matrix whose inverse gives a good approximation to the covariance matrix that enters into the model misfit calculation [8]. Recent commercial aids to history matching have been based on gradient techniques. Whereas the codes automatically generate a history-matched model, they do not guarantee that it is the correct global optimum solution. This realization has led to a new approach to the problem of history matching namely, the use of stochastic methods.

Prominent among the stochastic methods are those based on simulated annealing and genetic algorithms, see e.g., $[11,13]$. In general, these algorithms have slower convergence rates, compared with deterministic algorithms. Further, even though several realizations of the model are generated at each stage, there is limited and selective use of information from previously generated models.

The Neighbourhood Approximation (NA) sampling algorithm is a stochastic algorithm, which falls in the same category as genetic algorithms and simulated annealing. This paper presents the use of the algorithm in generating multiple history-matching models. The Neighbourhood Approximation algorithm firstly identifies all good history matching regions of the parameter space. It then generates an ensemble of models that preferentially sample these regions of the model parameter-space in a guided way, by using all information obtained about the model space. This approach overcomes one of the principal weaknesses of stochastic algorithms namely, slow convergence. It uses Voronoi cells to represent the parameter space.

\section{Voronoi cells and the Neighbourhood Approximation algorithm}

Given $n$ points in a plane, their Voronoi diagram divides the plane according to the nearest neighbor rule namely, each point is associated with the region of the plane closest to it. The distance metric is usually Euclidean, and each Voronoi polygon in the Voronoi diagram is defined by a generator point and a nearest neighbor region, see Figure $1 . \mathbf{a}$. 
Voronoi cells possess very attractive geometrical and computational properties, see [7]. Figure 1.b. shows the Voronoi diagram for 100 points in space. As new points are added, the Voronoi diagram is updated based on the nearest neighbor.rule. Figure 1.c. shows the updated diagram when 200 new points are added to the plane (Observe rectangular region ABCD). Thus Voronoi cells are unique (non-overlapping), space filling, and have size (volume, area) inversely proportional to the density of the generating point.

The Neighbourhood Approximation (NA) algorithm was originally developed to solve an inverse problem in earthquake seismology [14]. It differs from other stochastic algorithms in the following way. Rather than seeking a global optimal, the objective is to generate an ensemble of data-fitting models. The algorithm works by first, representing the parameter space with Voronoi cells. By constructing an approximate misfit surface for which the forward problem has been solved, the algorithm samples the parameter space by doing non-linear interpolation in multidimensional space, exploiting the neighborhood property of the Voronoi cells. The sampling is done in a guided way such that new realizations of the model are concentrated in regions of parameter space that give good fit to the observed data. See [14] for detailed description of the algorithm, and [2,18] for an earlier application in petroleum engineering.

Specifically, the NA-algorithm generates multiple data-fitting models in parameter space in the following way. Firstly an initial set of $n_{s}$ models are randomly generated. In the second step, the $n_{r}$ models with the lowest misfit models among the most recently generated $n_{s}$ models (including all previously generated models) are determined. Finally, new $n_{s}$ models are generated by uniform random walk in the Voronoi cell of each of the $n_{r}$ chosen models. The algorithm returns to the second step and the process is repeated. At each iteration $n_{r}$ cells are resampled and in each Voronoi cell, $n_{s} / n_{r}$ models are generated. The performance of the algorithm depends on the ratio $n_{s} / n_{r}$, rather than on the individual tuning parameters.

We note that at any stage of the sampling procedure, selective sampling of good data-fit regions is achieved by exploiting information about all previously generated models. In this way it attempts to overcome a main concern of stochastic sampling - poor convergence.

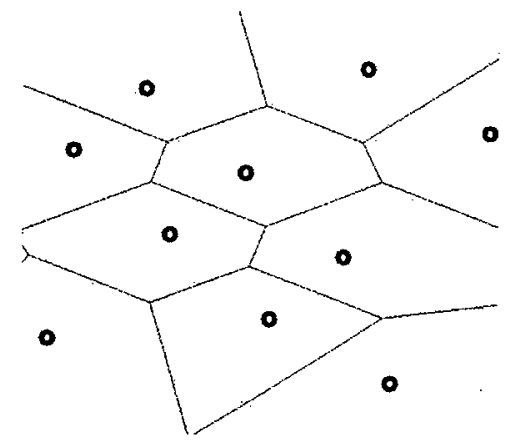

a. Random points in space

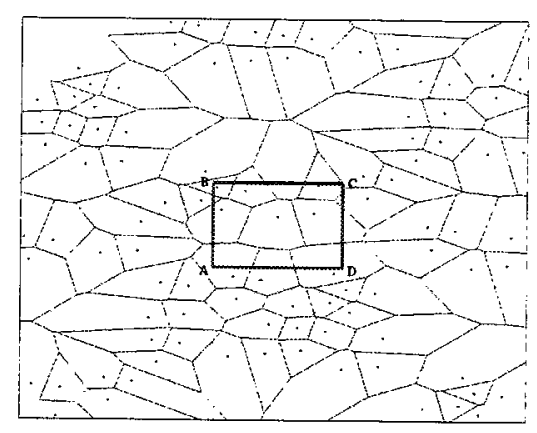

b. 100 points

Figure 1. Voronoi diagram

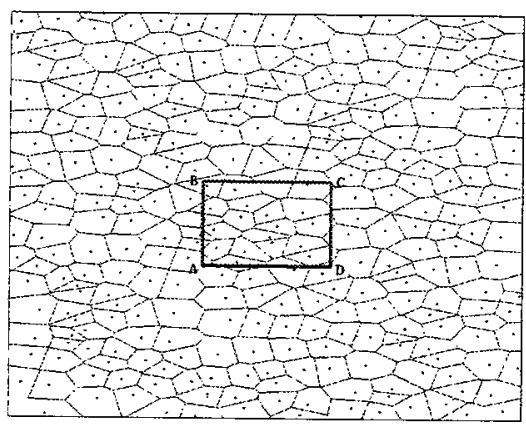

c. Update of b. -300 points

\section{Appraising the ensemble Sampling from the posterior}

Prior knowledge of each model $m$ in the ensemble can be probabilistically expressed through a prior probability density function $p(m)$. Our prior beliefs change as we compare the model output with the observed data. This comparison allows us to quantify the likelihood that the observed data is explainable by the model for which the likelihood holds. Bayes theorem allows us to update our prior beliefs by calculating a posterior probability $p\left(m \mid \Gamma_{h}\right)$, using the likelihood $p\left(\Gamma_{h} \mid m\right)$. By Bayes theorem, the posterior probability is defined by 


$$
p\left(m \mid \Gamma_{h}\right)=\left[p\left(\Gamma_{h} \mid m\right) p(m)\right] / \int p\left(\Gamma_{h} \mid m\right) p(m) d m
$$

The benefit of a Bayesian approach over other traditional methods of uncertainty analysis is that it permits the use of arbitrary probability distributions, not just Gaussian distributions, and of arbitrary measures of uncertainty, not just variance. It also extends the analysis to higher levels of interpretation, e.g., the rejection of any particular model, and the selection of appropriate models $[7,17]$. However, one of the challenges in applying Bayes theorem lies in evaluating the integral expression i.e., the normalizing term. This task is usually non-trivial, even for problems of very low dimension. However, Markov Chain Monte Carlo (MCMC) provides a method for generating a sequence of realizations that are samples from the posterior distribution without the need to calculate this term. The NA-Bayesian algorithm uses a MCMC approach to generate new models whose distribution approximates that of the input ensemble. For details of the NA-Bayesian algorithm, see [15].

\section{Application Model and problem description}

This paper presents an application of the algorithm to synthetic data. We use a fine grid model to generate synthetic data and history match this data, for a limited time, using a coarser model.

The fine grid is the SPE $10^{\text {th }}$ Comparison Solution Project model [3]. It is a geostatistical model defined on a three dimensional domain $[(x, y), z] R^{2} \times R$, being [1200x2200x170] cubic feet, and discretized into [60 $220 \times 85]$ cells. The top $70 \mathrm{ft}$ represents the Tarbert formation, while the bottom 100ft is the Upper Ness. There are 4 producer wells in the corners of the model at constant BHP, and a central water injector at constant injection rate. There are two phases, oil and water, and the relative permeability curves are of Corey type, i.e.,

$$
\begin{aligned}
K_{r o}(S) & =\left(\left[S-S_{w c}\right] /\left[1-S_{w c}-S_{o r}\right]\right)^{2} \\
K_{r w}(S) & =\left(\left[1-S-S_{o r}\right]\left[\left[1-S_{w c}-S_{o r}\right]\right)^{2}\right. \\
S_{w c} & =S_{o r}=0.2 .
\end{aligned}
$$

The coarse grid is a single-phase scale-up of the fine grid, and consists of $[5 \times 11 \times 10]$ cells. The well positions and controls are identical to the fine grid model. The coarse model relative permeability curves were defined using functions by [1], and expressed by

$$
\begin{aligned}
K_{r o}(S) & =K_{r o}\left(S_{i w}\right) \exp \left(\alpha R^{-\varsigma}\right), \\
K_{r w}(S) & =K_{r o}\left(S_{o r}\right) \exp \left(-\beta R^{-\eta}\right), \\
R & =\left(S-S_{i w}\right) /\left(1-S_{o r}-S\right) .
\end{aligned}
$$

The parameter space is thus defined by $p=\left(K_{r o}, \alpha, \varsigma, K_{r w}, \beta, \eta\right)$. The two distinct geological formations were modeled with separate pairs of relative permeability curves. Hence we solve a 12-dimensional parameter space problem. Firstly, we run the fine grid model to obtain fine grid average pressure and produced oil rate for 2000 days. We use data for 300 days as history data.

To create a more realistic case, we added uncorrelated random noise to the fine grid data in the following way. We drew a set of random numbers $r$ nd from a normal distribution rnd $N(0,1)$, and defined

$$
\text { Simulated field data }=\text { fine grid data }+\sigma_{d} \cdot \text { rnd }
$$

Figure 2 below shows the simulated field data, as well as the original fine grid data for the average pressure and oil rate.

The task is to use the coarse grid model and history match, by determining relative permeability curves. Finally, we forecast the fine grid behavior out to 2000 days, and quantify the uncertainty in our forecast. 


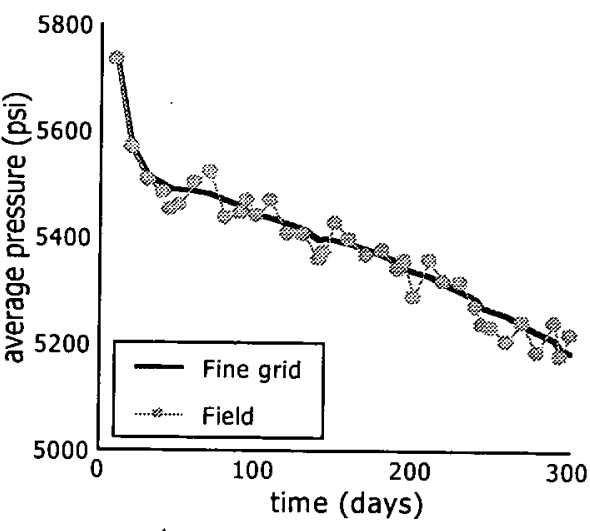

a. Average pressure

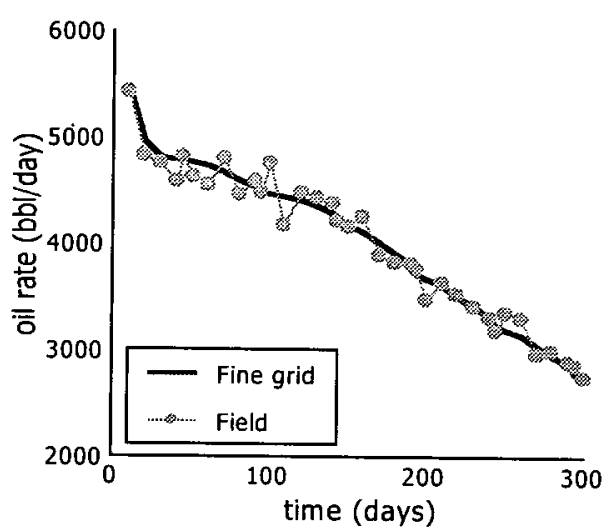

b. Oil rate

Figure 2. Simulated history data

\section{Quantifying misfit}

The two major sources of error affecting uncertainty in our predictions, and which need be accounted for are the model and observation errors. The data errors encapsulate lack of knowledge of the subsurface geology and errors inherent in the dynamic data. The model errors on the other hand depend on the choice of numerical simulator we choose, i.e., mass balance or streamline, finite element or finite difference. These errors arise from the approximation of the originally continuous conservation and flow equations with discrete analogues, and the inability to capture sub-grid details. The main error however, is due to the inability to capture sub-grid details. Consideration must be given to errors due to numerical diffusion and cell-aspect ratio [2]. Numerical diffusion means front resolution is impossible over fewer than three grid cells, while cell-aspect ratio errors are due to the sensitivity of simulation to cell height/thickness ratio. We note that simulation errors are correlated in time. This time correlation must therefore be included in assessing model fit to data otherwise acceptable models are rejected if too many points are included in the time series of production data. This would have a major impact on any scheme that required real time rate and pressure data.

We define an objective function with a structure that offers flexibility in combining data of different scales, which may be correlated or uncorrelated, and the inclusion of a priori information. In the least square sense, we define the measure of misfit [19], $\Omega$, as

$$
\Omega=\sum_{n_{w}}\left\langle\partial \vec{P}\left|C_{p}^{-1}\right| \partial \vec{P}\right\rangle+\sum_{n_{w}} \sum_{j=o, w}\left\langle\partial \vec{Q}_{j}\left|C_{j}^{-1}\right| \partial \vec{Q}_{j}\right\rangle
$$

In equation (7), $\partial \vec{P}$ and $\partial \vec{Q}$ refer to the difference between the simulated and fine grid data, for pressure and production rates, respectively. The matrices $C_{p}$ and $C_{j}$ are covariance matrices that capture both data and model errors, and $n_{w}$ refers to number of sets of data. The structure of the covariance matrices depends on the type of error models adopted. The use of error models is attractive because among other things, the approach is statistical. The functional forms, even though simple, give quantitative expressions, which show the dependence of the errors on parameters such as aspect ratios and correlation properties, and they are easy to calibrate, see [4, 5]. Different structures for the covariance matrix exist in the literature. Notably are the Gaussian, exponential, spherical and hole-effect model structures.

Here, we present results where the model covariance matrix structure is defined by an exponential model, which takes into account the time correlation of the errors: 


$$
C^{m}\left(t_{i}, t_{k}\right)=\sigma_{m}^{2} \exp \left(-\left|t_{i}-t_{k}\right| \tau^{-1}\right) .
$$

In equation (8) above, $\tau$ defines the lag i.e., the time interval over which the errors are assumed to be correlated, and $\sigma_{\mathrm{m}}$ is the variation expected in the model errors. We have taken $\tau=100$ days. Assuming no covariance in the data errors, we define the data covariance matrix by

$$
C^{d}\left(t_{i}, t_{k}\right)=\delta_{i k} \sigma_{d}^{2} \text {. }
$$

To illustrate, Figure 3 below gives an example of the covariance structures for the oil rate.

Given a point $m$ in model space, the likelihood that the observation $\Gamma_{h}$ can be obtained by $m$ is given by

$$
p\left(\Gamma_{h} \mid m\right) \sim \exp (-0.5 \Omega) .
$$

The refinement criterion for the Neighbourhood Approximation algorithm is based on the rank of the misfit $\ell$. This is defined as

$$
\ell=-\log \left(p\left(\Gamma_{h} \mid m\right)\right)=0.5 \Omega \text {. }
$$

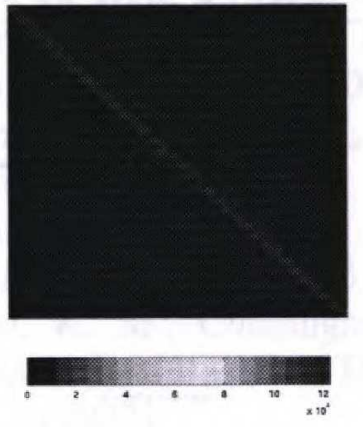

a. Data errors

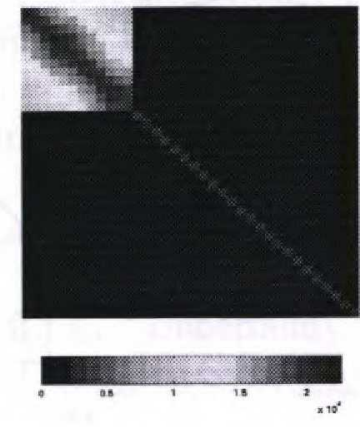

b. model errors

Figure 3. Covariance structures

\section{Results}

Quantifying uncertainty requires several hundreds to thousands of realizations. In this paper we presents results where we have used the NA-algorithm to generate 312 models by sampling a 12dimensional space. To quantify the uncertainty in our predictions, we run a long chain of the MCMC algorithm on the misfit surface and performed a Bayes update of the probabilities. We monitored the frequency of visits to each Voronoi cell during the random walk. Thus we are able to calculate the relative probability $w_{j}$ of each model $m_{j}$ in the ensemble of size $n_{m}$, based on the frequency $f_{j}$, using equation (12). Since the MCMC algorithm samples from the posterior distribution, the calculated probability is representative of the posterior probability of each model.

$$
w_{j}=f_{j} / \sum_{k=1}^{n_{m}} f_{k} .
$$

Assuming Gaussian statistics, we calculate the first and second moments of the pressure and production profiles by

$$
\begin{aligned}
\langle Q(t)\rangle_{1} & =\sum_{k} w_{k} Q_{k}(t) . \\
\langle Q(t)\rangle_{2} & =\sum_{k} w_{k} Q_{k}^{2}(t)-\langle Q(t)\rangle_{l}^{2} .
\end{aligned}
$$

Using these parameters, we determined $\mathrm{P}_{10}$ and $\mathrm{P}_{90}$ cut-off for each of the predicted profiles. Figure 4 shows plots of the 1-dimensional posterior probability distribution for each of the parameters. These are projections of the multidimensional posterior probability distribution onto each of the 12-parameter axes. 
Figure 5 shows plots of the maximum likelihood prediction, the average, and the $P_{10}-P_{90}$ cutoff profiles for the oil and water rates, and the field average pressure. We observe that the $P_{10}-$ $P_{90}$ profiles envelope the maximum likelihood prediction as well as the fine grid data.
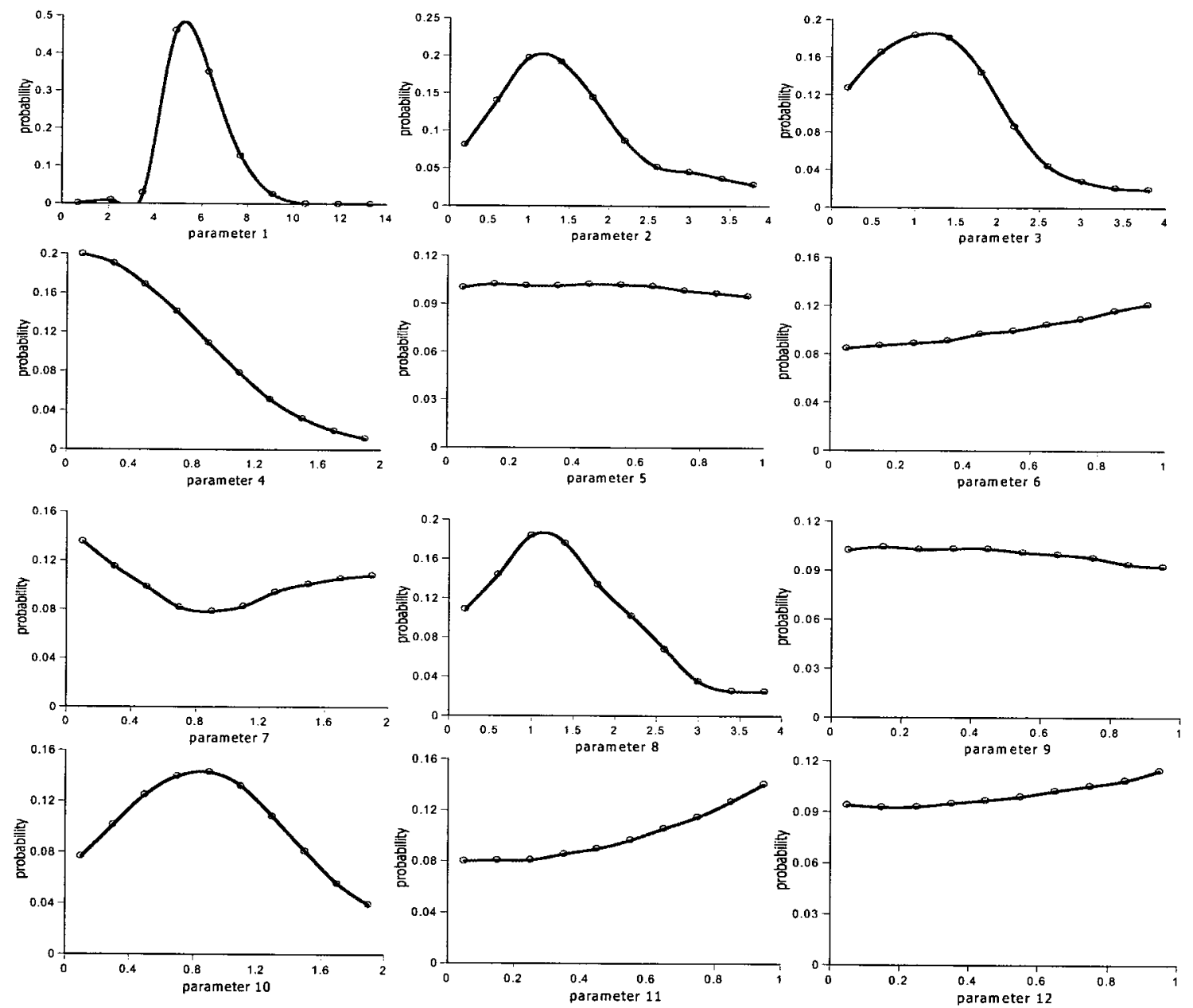

Figure 4. 1D marginal probability plots (parameters 1-12)
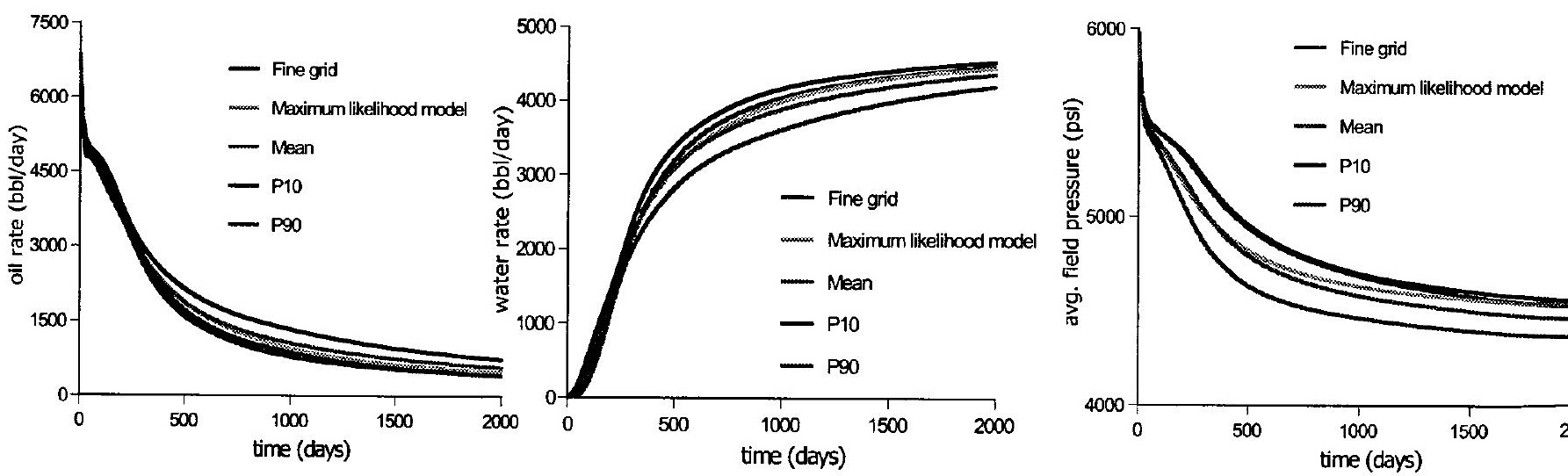

Figure 5. Uncertainty envelops for oil, water and pressure profiles

\section{Conclusions}

This paper has demonstrated an approach for generating uncertain history matching models, and quantifying uncertainty in model performance prediction using the Neighbourhood Approximation algorithm. The approach is able to incorporate both data and model errors in quantifying the degree of model fit to the observed data, and in defining the model likelihood. 
We have demonstrated the strength of the algorithm using a benchmark data set for which the true solution is known. Our results show that in this case the maximum likelihood model is a good forward predictor. The uncertainty bounds computed encounter the true solution.

To quantify uncertainty in model predictions, the Neighbourhood algorithm was employed in a Bayesian framework and that the true solution lies within the uncertainty bounds predicted by the algorithm.

\section{References}

1. Chierici, G., "Novel Relations for Drainage and Imbibition Relative Permeabilities" , (SPE 10165)

2. Christie, M., MacBeth, C., and Subbey, S., "Multiple history-match models for Teal South", (The Leading Edge, 2002)

3. Christie, M. A., and Blunt, M. J., "Tenth SPE Comparative Solution Project: A Comparison of Upscaling Techniques", (SPE 66599)

4. Glimm, J., Hou, S., Kim, H., and Sharp, D. H., "A Probability Model for Errors in the Numerical Solutions of a Partial Differential Equation". Computational Fluid Dynamics Journal, Vol. 9, 485-493 (2001).

5. Glimm, J., Hou, S., Lee, Y., and Sharp, D. H., "Prediction of Oil Production with Confidence Intervals", (SPE 66350).

6. Hadamard, J., Lectures on the Cauchy Problem in Linear Partial Differential Equations, Yale University Press, 1923.

7. Hansen, K. M., Cunningham, G. S., and McKee, R. J., "Uncertainty Assessment for Reconstructions Based on Deformable Geometry", Int. J. Imaging Syst. Technology, Vol. 8, 506-512 (1997)

8. Lépine, O. J., et al., "Uncertainty Analysis in Predictive Reservoir Simulation Using Gradient Information', SPEJ, Vol.4, Nr. 3, (1999)

9. Okabe, A., Boots, B., and Sugihara, K., 'Spatial Tesselations - Concepts and Applications of Voronoi diagrams', John-Wiley \& Sons, England, 1992.

10. Oliver, D. S., Cunha, L. B. and Reynolds, A. C., "Markov chain Monte Carlo methods for conditioning a permeability field to pressure data", Mathematical Geology, Vol. 29, Nr. 1, 61-91 (1997)

11. Portella, R. C. M .and Prais, F. "Use of Automatic History Matching and Geostatistical Simulation to Improve Production Forecast", (SPE 53976)

12. Roggero, F., "Direct Selection of Stochastic Model Realizations Constrained to Hsitory Data", (SPE 38731)

13. Romero, C. E., Carter, J. N., Zimmerman, R. W. and Gringarten, A. C., "Improved reservoir characterization through evolutionary computation", (SPE 62942)

14. Sambridge, M., "Geophysical inversion with a Neighbourhood Algorithm, Part 1: Searching a parameter space", (Geophysical Journal International, 1999a)

15. Sambridge, M., "Geophysical inversion with a Neighbourhood Algorithm, Part 2: Appraising the ensemble", (Geophysical Journal International, 1999b)

16. Schulze-Riegert, R. W., Axmann, J. K., Haase, O., Rian, D. T. and You, Y.-L., "Optimization methods for history matching of complex reservoirs", (SPE 66393)

17. Sivia, D. S., 'Data Analysis - A Bayesian Tutorial', Claredon Press, Oxford, 1996

18. Subbey, S., Christie, M., and Sambridge, M., "Uncertainty Reduction in Reservoir Modeling", (AMS Con. Maths. Series) - In press

19. Tarantola, A., Inverse Problem Theory, Methods for Data Fitting and Model Parameter Estimation, Elsevier Scinece Publishers, Amsterdam, 1987. 